\title{
First Steps Towards Process Mining in Distributed Health Information Systems
}

\author{
Emmanuel Helm and Ferdinand Paster
}

\begin{abstract}
Business Intelligence approaches such as process mining can be applied to the healthcare domain in order to gain insight into the complex processes taking place. Disclosing asis processes helps identify room for improvement and answers questions from medical professionals. Existing approaches are based on proprietary log data as input for mining algorithms. Integrating the Healthcare Enterprise (IHE) defines in its Audit Trail and Node Authentication (ATNA) profile how real-world events must be recorded. Since IHE is used by many healthcare providers throughout the world, an extensive amount of log data is produced. In our research we investigate if audit trails, generated from an IHE test system, carry enough content to successfully apply process mining techniques. Furthermore we assess the quality of the recorded events in accordance with the maturity level scoring system. A simplified simulation of the organizational workflow in a radiological practice is presented. Based on this simulation a process miing task is conducted.
\end{abstract}

Keywords-audit trail and node authentication, extensible event stream, integrating the healthcare enterprise, process mining, RFC-3881, digital imaging and communications in medicine

\section{INTRODUCTION}

$\mathbf{P}$ ROCESS mining is an emerging discipline based on existing data mining techniques that also takes the complexity of the underlying business processes into account. By deriving process models from observed system behavior (i.e. event logs) process mining is able to provide understanding of the as-is processes [1], [2].

[1] distinguish between three types of process mining approaches (see Fig. 1). Discovery produces a model from an event $\log$ without a-priori information. Conformance can be used to check if the recorded reality in a log conforms to a predefined model. Enhancement adds a feedback loop to the conformance checking, aimed at model improvement.

In the last years, tool support for process mining increased steadily. Tools like the open-source proM framework [3] or Disco from Fluxicon [4] offer a wide range of approaches to process mining. Disco supports only process discovery, whereas proM also provides plugins for conformance checking and process enhancement.

The findings of several research initiatives already propose the use of process mining to extract information from event logs in healthcare [5], [6], [7], [8], [9]. Some aim to assess how those systems are used or misused, others try to gain clinical knowledge from data or to improve the quality of hospital workflows. The event data used in these approaches originated

E. Helm and F. Paster are with the Research Department of e-Health, Integrated Care, University of Applied Sciences Upper Austria, Austria, 4232, Hagenberg (e-mail: emmanuel.helm@fh-hagenberg.at; ferdinand.paster@fhhagenberg.at) from various different sources such as medical devices or department specific or hospital information systems. For every source, the event data needs to be preprocessed to meet the requirements of process mining tools.

\section{A. Standardized Auditing}

Integrating the Healthcare Enterprise (IHE) is an international initiative by healthcare professionals and industry to improve the integration and interoperability of Health Information Systems (HIS). It started in 1998 as an initiative to define how existing standards, like Digital Imaging and Communications in Medicine (DICOM) and Health Level Seven (HL7), can be implemented to overcome common interoperability problems in radiology [10]. Currently the IHE integration profiles are the basis for HIS of major vendors [11], national healthcare programs like the Austrian electronic health record (ELektronische GesundheitsAkte - ELGA) [12], the Smart Open Services for European Patients (epSOS) project [13] and the transatlantic Trillium Bridge project [14].

One of the basic IHE Integration Profiles, the Audit Trail and Node Authentication (ATNA) Profile, defines how to build up a secure domain that provides patient information confidentiality, data integrity and user accountability. A secure domain can scale from department, to enterprise or crossenterprise size. To ensure user accountability, ATNA specifies the use of a centralized Audit Record Repository (ARR) where all audit messages are stored. Consequently violations of security policies can be detected, especially regarding Protected Health Information (PHI) which includes all kinds of patientidentifiable information records [15].

\section{B. Querying an Event Log}

The ATNA Profile defines how event data should be collected within (distributed) HIS and states four questions that must be answerable based on the information in an ARR [16]:

- "For some user: which patients' PHI was accessed?"

- "For some patient PHI: which users accessed it?"

- "What user authentication failures were reported?"

- "What node authentication failures were reported?"

Depending on the physical representation of the ARR (log file, SQL database, NoSQL database, etc.) the four questions can be answered, e.g. utilizing a SQL query or running a simple Perl script. However there are no mechanisms described to answer more sophisticated questions like:

- "What are the typical clinical pathways in our hospital?"

- "Which medical departments collaborate frequently?"

- "Where are the bottlenecks in our clinical pathways?" 


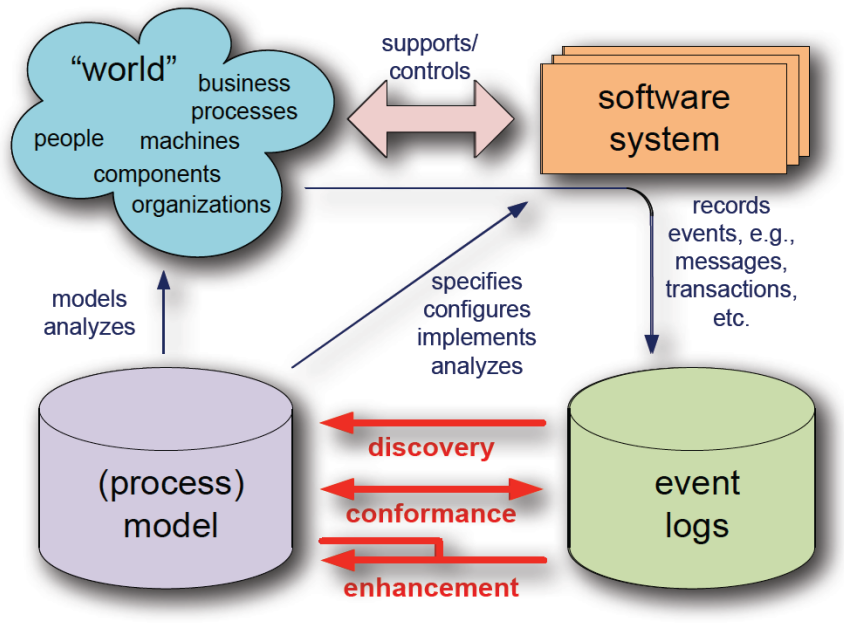

Fig. 1. Positioning of the three main types of process mining: (a) discovery, (b) conformance checking, and (c) enhancement [1]

This work assumes that the answers to these and other questions are implicitly contained in the ARR's data and a process mining based approach could be utilized to discover this information. The goal of this research is to find a way to enable process mining in distributed health information systems, without having to deal with an increasing preprocessing effort. The core question is: Does log-data, produced by means of the standardized ATNA Integration Profile, provide sufficient information to apply process mining methods?

\section{Methods}

Bozkaya, Joost, and Werf describe a general approach to process discovery called Process Diagnostics [17]. It comprises five phases that can be organized in three steps:

1) Preprocessing: In the first phase log preparation the log has to be preprocessed in order to use it for process mining. This includes semantic analysis so that the activities, events and timestamps can be mapped correctly to a standardized process mining format like the one described in II-C. Log inspection is about getting a deeper understanding about what is going on in the log. Statistics about the absolute number of cases, filesize, events per case, roles, etc. are collected. It also represents the second stage of preprocessing, where incomplete cases are removed.

Preprocessing of the log, the preparation for further process mining steps, is a complex task that already raises many questions [17]. This paper presents an approach aimed to prepare an IHE compliant ARR for further analysis by the means of process mining, i.e. discovery.

2) Analysis: The three aspects of analysis - control-flow, performance and role - describe how to extract new information from the preprocessed log. Different algorithms and techniques are available, for example to visualize the as-is processes, to find bottlenecks or to identify involved actors [2].

3) Transfer Results: The last step is suitable for the adherence of other types of process mining. The performer of the discovery is usually not able to make the distinction between

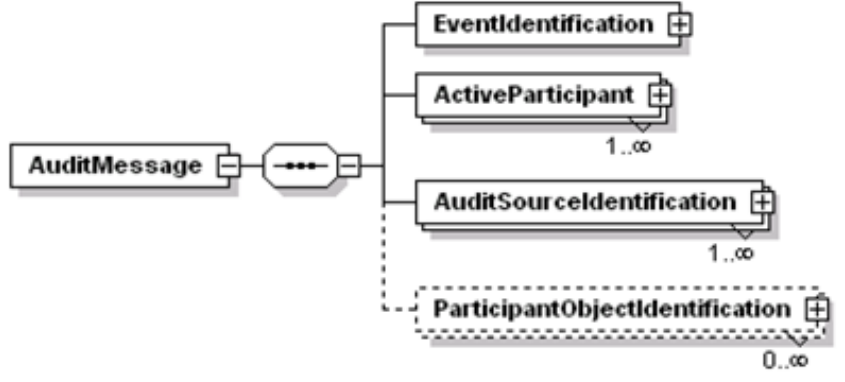

Fig. 2. Schema diagram for audit messages defined by IHE based on RFC3881 and DICOM [15].

TABLE I

SElECTED RFC-3881 FiElds. 1-6 ARE MANDATORY ACCORding to [16]. 7-8 ARE MANDATORY IF THE ParticipantObjectIdentification IS PRESENT.

\begin{tabular}{cll}
\hline $\mathrm{Nr}$ & Name & Location in Schema \\
\hline \hline 1 & EventID & EventIdentification \\
\hline 2 & EventDateTime & EventIdentification \\
\hline 3 & EventOutcomeIndicator & EventIdentification \\
\hline 4 & UserID & ActiveParticipant \\
\hline 5 & UserIsRequestor & ActiveParticipant \\
\hline 6 & AuditSourceID & AuditSourceIdentification \\
\hline 7 & ParticipantObjectID & ParticipantObjectIdentification \\
\hline 8 & ParticipantObjectIDTypeCode & ParticipantObjectIdentification \\
\hline
\end{tabular}

wanted and unwanted behavior. Therefore it is important to discuss the outcome with the client, so that the client gets a better understanding of the information system. Conformance checking and process enhancement are logical subsequent operations.

\section{A. ATNA Logs}

To map real-world activities to event logs, ATNA makes use of the Security Audit and Access Accountability Message XML Data Definitions for Healthcare Applications (RFC3881). It incorporates the viewpoints of different organizations like HL7, IHE, DICOM, ASTM and the NEMA/COCIR/JIRA Security and Privacy Committee [18].

DICOM standardized parts of the RFC-3881 vocabulary and defined additional and optional elements [16]. IHE specifies the use of the DICOM vocabulary and provides extensions. Events that cannot be defined by the basis of the DICOM vocabulary have to be reported using the more general RFC3881 schema. Events that cannot be described by that schema cannot be reported to an ARR [15]. Fig. 2 shows the respective schema diagram for audit messages.

\section{B. Audit Message Semantics}

The content of an IHE Audit Message heavily depends on the type of action performed. Since Audit Messages have to capture a broad range of different events happening in an IHE environment, the format is modular and audit logs can be diverse. 
According to DICOM the fields 1-6 in table I are mandatory, whereas 7-8 are mandatory only in context of the ParticipantObjectIdentification section which is optional as whole. As shown in Fig. 2 the IHE model conforms to that specification. However, we checked the audit logs of two independent IHEcompliant systems and found that the ParticipantObjectIdentification is usally recorded.

In the RFC-3881 the mandatory fields are described in [18] as follows:

1) EventID: "Identifier for a specific audited event, e.g., a menu item, program, rule, policy, function code, application name, or URL. It identifies the performed function."

2) EventDateTime: "Universal coordinated time (UTC), i.e., a date/time specification that is unambiguous as to local time zones."

3) EventOutcomeIndicator: "Indicates whether the event succeeded or failed."

4) UserID: "Unique identifier for the user actively participating in the event."

5) UserIsRequestor: "Indicator that the user is or is not the requestor, or initiator, for the event being audited."

6) AuditSourceID: "Identifier of the source where the event originated."

7) ParticipantObjectID: "Identifies a specific instance of the participant object."

8) ParticipantObjectIDTypeCode: "Describes the identifier that is contained in Participant Object ID."

\section{Standardized Event Log Representation}

Log data is created from a variety of different systems with their own proprietary data format and semantics. Because log data is the key input for process mining, a standardized data format for the event logs is needed.

In the past the Mining eXtensible Markup Language (MXML) was used as an XML-based format for log exchange. To overcome the limitations of MXML, concerning primarily extensibility, eXtensible Event Stream (XES) was developed [2]. In September 2010 the IEEE Task Force on Process Mining accepted XES as standard for log data exchange [19].

XES defines three basic objects (see Fig. 3): Log, Trace and Event. Log (the process) contains a collection of Traces (execution instances) and a Trace contains a collection of Events. Each object can contain an arbitrary set of strongly typed attributes. Every attribute has a type, like String, Boolean and Date. To add semantics to these data types, XES defines the concept of extensions. An extension dictates a set of attributes, their type and keys with a specific semantic meaning.

For improved mutual understanding, standard extensions were defined, e.g. Concept, Organizational, Time, etc. For example the Time extension defines a timestamp attribute. Using these standard extensions yields the benefit that process mining tools like ProM, Disco, etc. can semantically interpret the given data [3].

To define mandatory fields in XES, so called global attributes can be used. For example if Event is defined to have certain global attributes, like a timestamp or resource information, all events in the log must contain those.

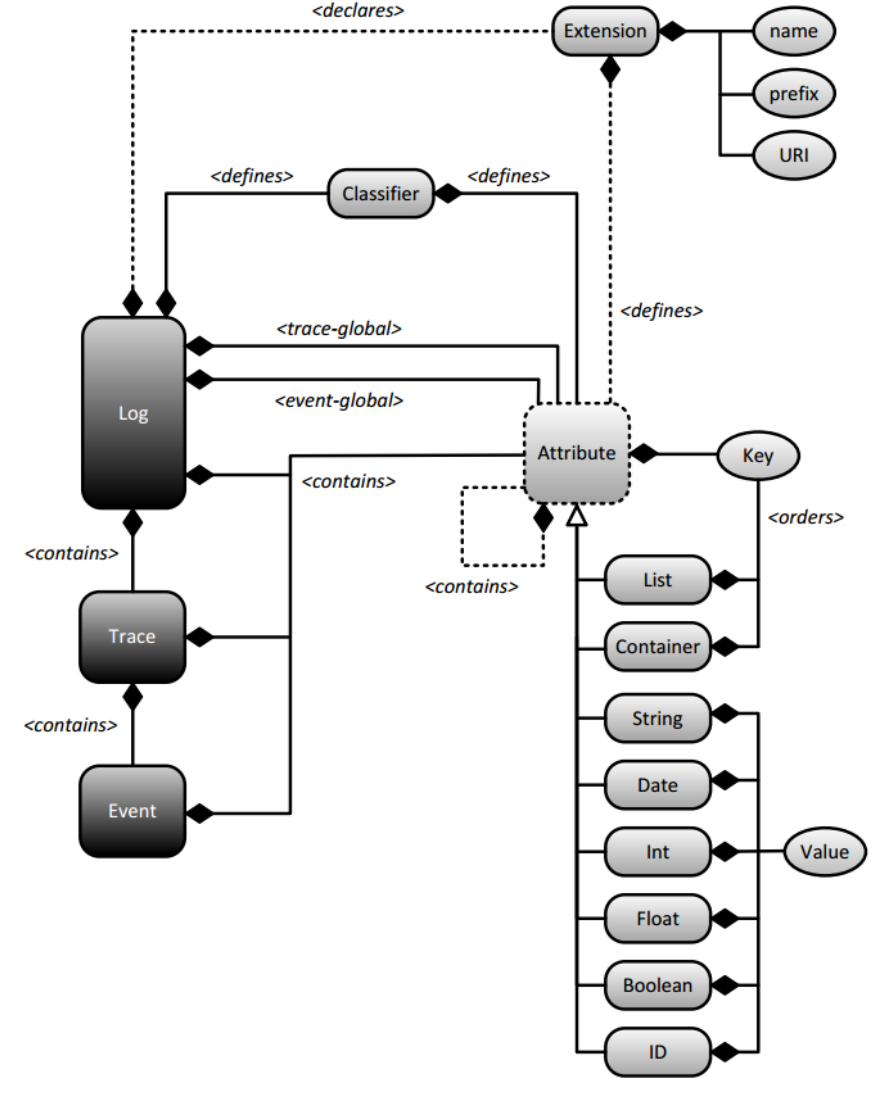

Fig. 3. The XES meta-model [19]

\section{Transformation of the Log}

Process mining tools like Disco or proM provide import interfaces that allow to map certain fields of a database or a CSV-file to the respective XES fields. The mapping strongly depends on the current process mining task and the questions that should be answered. For example proM includes the XESame application to support the import of non-event $\log$ databases [3]. Disco also allows the import of comma separated text files. In both cases the mapping task must be carried out manually, otherwise you can not import the data.

The goal of the transformation approach was to keep as much information as possible and provide an automatic but semantically correct mapping from an ATNA log to an XES event $\log$ [20]. Thus making it possible to first conduct process mining tasks and then decide how to continue processing the $\log$ to answer more specific questions like the ones stated in I-B.

In order to convert an event log recorded by the means of ATNA into an XES event log, we developed a transformation architecture based on the Meta Object Facility (MOF) standard [21]. It is influenced by the Model Driven Interoperability (MDI) approach in [22].

Fig. 4 shows the hierarchy of models [20]. XML serves as the meta-meta-model for RFC-3881 and XES. Between the two meta-models at $\mathrm{M} 2$ a definition exists how to map the components of RFC-3881 on XES. At M1, the specific instance of the RFC-3881 model, the Audit Trail, is transformed into a specific instance of the XES model, the Mining Log. 


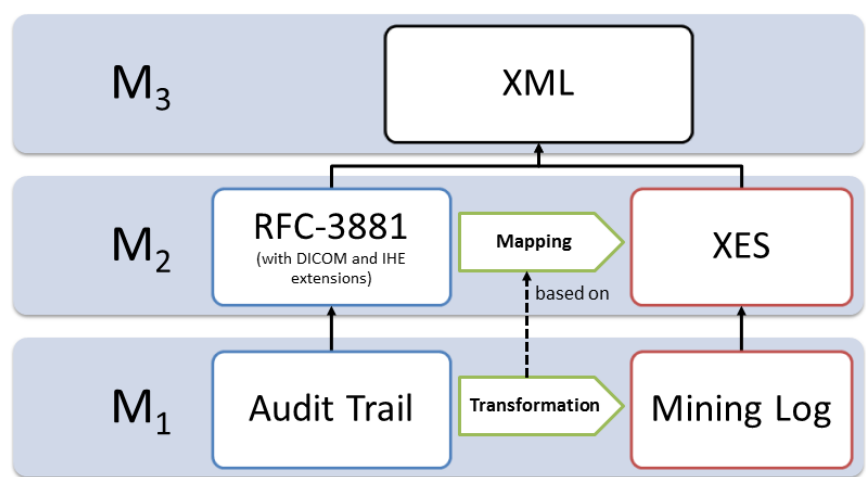

Fig. 4. Transformation architecture to convert RFC-3881 based Audit Trails into standardized XES Mining Logs [20].

Both models on the M1 layer conform to their respective metamodel. Of course, according to the MOF standard, the Audit Trail is also just a model representing the actual real-world events on M0.

In case of a log file the actual transformation of the Audit Trail into the Mining Log is conducted via Extensible Stylesheet Language Transformations (XSLT). The mapping on M2 represents a Model-to-Model (M2M) transformation, thus enabling an automatic transformation of model instances an M1.

\section{E. Building a Test System}

For the creation of audit messages an IHE test system, based on the OpenHealthTools, was utilized [23]. This environment was initially set up on behalf of the research project Workflow for Image prefetching in Radiology for ELGA (WIRE) with the aim of testing different prefetching mechanisms for radiological image data.

The system records the audit messages sent by IHE actors executing transactions. The test environment was designed in analogy to parts of the planned ELGA infrastructure. This yields the benefit of being able to analyze auditing conditions and information content within the scope of a nationwide implementation of an IHE based Electronic Health Record (EHR).

Relevant actors, cf. Fig. 5, originate from the IHE profiles Cross-Enterprise Document Sharing (XDS), Patient Identifier Cross Referencing (PIX) and Patient Demographics Query (PDQ). Imaging data from CTs, MRIs etc. is stored in a Picture Archiving and Communication System (PACS).

Some transactions that are frequently executed in this test system:

- ITI-9, PIX Query, to query patient identifiers.

- ITI-29, PDQ Query, to query a patient's demographic data.

- ITI-41, XDS Provide and Register Document Set

- ITI-18, XDS Registry Stored Query, to search for a patient's data.

- ITI-43, XDS Retrieve Document Set, to load a patient's data.

Any transaction between actors is bilaterally recorded and saved in the ARR. I.e. the same action is recorded twice.

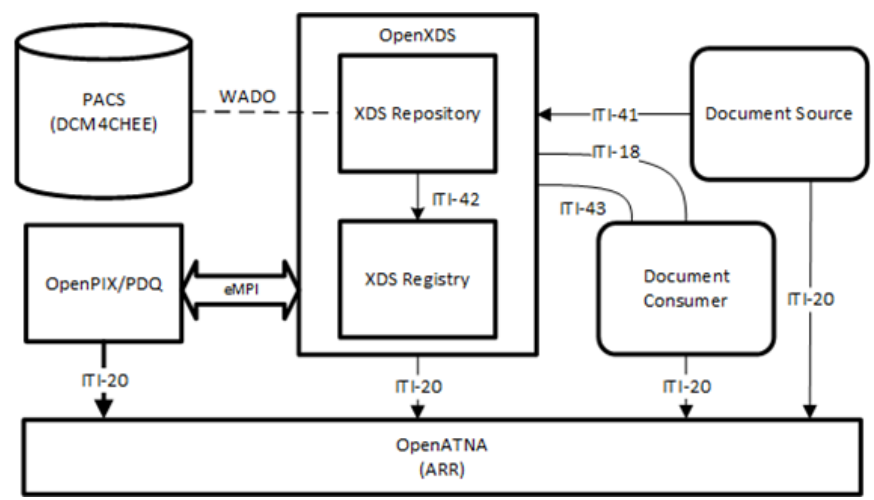

Fig. 5. The test system representing a distributed health information system in radiology. Patient management is handled by the OpenPIX/PDQ component The OpenXDS component handles the document management. All audit trails are recorded by the underlying OpenATNA ARR. Not all involved transactions are outlined.

\section{EVALUATION}

In course of the WIRE project the organizational workflows of three radiologists were analyzed and seven major steps were identified. Fig. 6 shows the process, modeled by the means of the Business Process Model and Notation (BPMN). It was simulated using the IHE actors and transactions implemented in the test system.

To relate the recorded events to the different steps of the process, post-processing of the transformed log was necessary. Since the process that produced the log was already known, the classification of the events was less complex. The EventID, EventActionCode and the EventTypeCode (mandatory for most transactions) mapped to the fields of the XES Concept extension were used to classify the events:

- Appointment and patient submission were associated with PIX and PDQ transactions, querying, updating or writing patient data. For example the IHE transaction ITI-9 was classified as the patient submission step.

- The examination led to the registration of a DICOM Key Object Selection (KOS) Document referring to the images in the PACS and the PACS recorded a DICOM Instances Transferred event (EventID: 110104, no EventTypeCode).

- Diagnosis started with the retrieval of the current images and previous reports and resulted in the registration of an audio file (EventID: 110106, EventTypeCode: ITI-41).

- In the report step the audio file was retrieved and a report was registered (EventID: 110107, EventTypeCode: ITI43).

- For the attestation only the XDS metadata of the report were changed - a legalAuthenticator was added (EventID: 110106, EventTypeCode: ITI-41, EventActionCode: "U"' Update).

- For the report transmission we assumed that the report and the images were handed to the patient, thus triggering an export audit event (EventID: 110106, no EventTypeCode, EventActionCode: "R" Read).

There are more potential classifiers and the post-processing has to be adapted to the specific process mining task. In this 


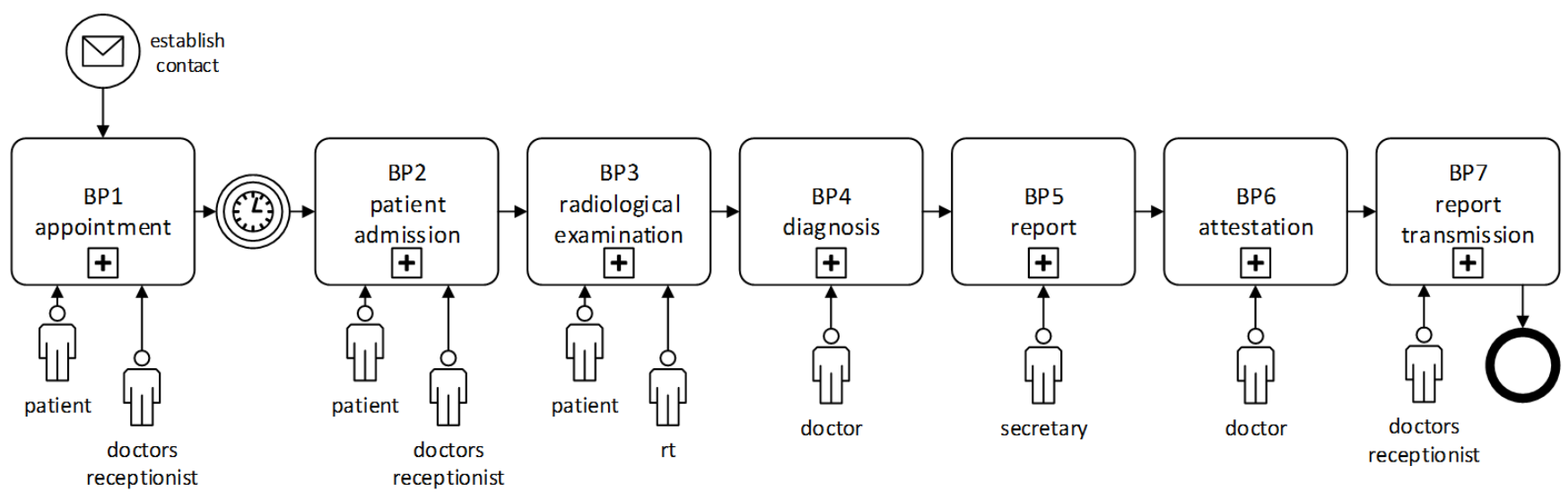

Fig. 6. BPMN model of the radiological workflow from an organizational point of view. The process starts with the patient arranging an appointment. At the appointed date the patient arrives at the clinic and registers for the examination. This patient admission is handled again by the doctor's receptionist and includes the handover of the referral. The results of the examination are saved in the local PACS. For the diagnosis the current examination results and prefetched previous findings are loaded. Based on this information the radiologist takes a dictation of the clinical findings. To create the report a secretary either listens to the dictation and writes it down or corrects the automated speech-to-text software. To finish the record the radiologist has to check and attest it. The final step is to transmit the report to the referring physician.

case the goal was to reconstruct a process looking like the one in Fig 6. After filtering the relevant events the process mining tool proM was used to visualize the result with the AlphaMiner plugin to generate a Petri net from the event log. This plugin uses the Alpha-algorithm that was first presented in [24].

\section{RESULTS}

The evaluation showed that the recorded audit trails provided sufficient information to allow a correct reconstruction of the radiological workflow. The resulting Petri net depicts the process steps from Fig. 6 using places and transitions. In Fig. 7 a detail of the Petri net shows the transitions ITI-41_U and 110106_R.

The left place corresponds to the patient waiting for the images and the report to be printed or burned on a CD. The right place marks the end of the process. Afterwards the patient leaves the clinic. ITI-41_U marks a metadata update operation indicating the attestation of an existing report. 110106_R marks a DICOM export operation indicating the report transmission.

\section{CONCLUSION}

Given the results the conclusion is that event logs recorded by the means of the ATNA integration profile provide enough information to facilitate process mining. This conclusion is reached by analyzing the source format, the target format and the transformation between them.

The presented approach was developed and tested in a lab environment. The scope of the test case was set to check the general applicability of ATNA logs for process mining. A higher effort regarding preprocessing, mapping and analysis will be required to mine logs recorded by big (distributed) HIS.

\section{A. Quality of the Log}

To define the applicability of a data source for process mining, [1] introduces different maturity levels for event logs based on the following quality criteria:

- Event $\log$ s should be trustworthy, i.e., it should be safe to assume that the recorded events actually happened and that the attributes of events are correct.

- Event $\operatorname{logs}$ should be complete, i.e., given a particular scope, no events may be missing.

- Any recorded event should have well-defined semantics.

- Moreover, the event data should be safe, i.e. privacy and security concerns are addressed while recording the events.

The first level describes event logs of poor quality, where recorded events may not correspond to reality and events may be missing. Examples for level 1 event logs are paper documents routed through the organization or paper-based medical records. The highest level of maturity can only be reached by fully integrated, semantically annotated logs of business process management systems.

Based on the classification system described in [1] we classify the ATNA $\log$ as level 3. There is an automatic recording mechanism and recorded events do match reality. It does not qualify for level 4 because of the lack of explicit notations of process instances and activities. Still the recorded event $\log$ exceeds the criteria for level 2 as the completeness is guaranteed and it is not possible to bypass the information system. Additionally the semantics of the recorded audit messages are well-defined as all fields in the messages have to be filled according to vocabularies defined in the RFC-3881 and DICOM standards [15].

[25] and [26] point out a big issue regarding data quality in HIS. The low granularity of timestamps, only the day of the event is recorded in some systems, consequently leads to problems identifying the correct order of events. In our approach all ATNA audit messages provide timestamps with very high granularity. This is ensured because the systems in 

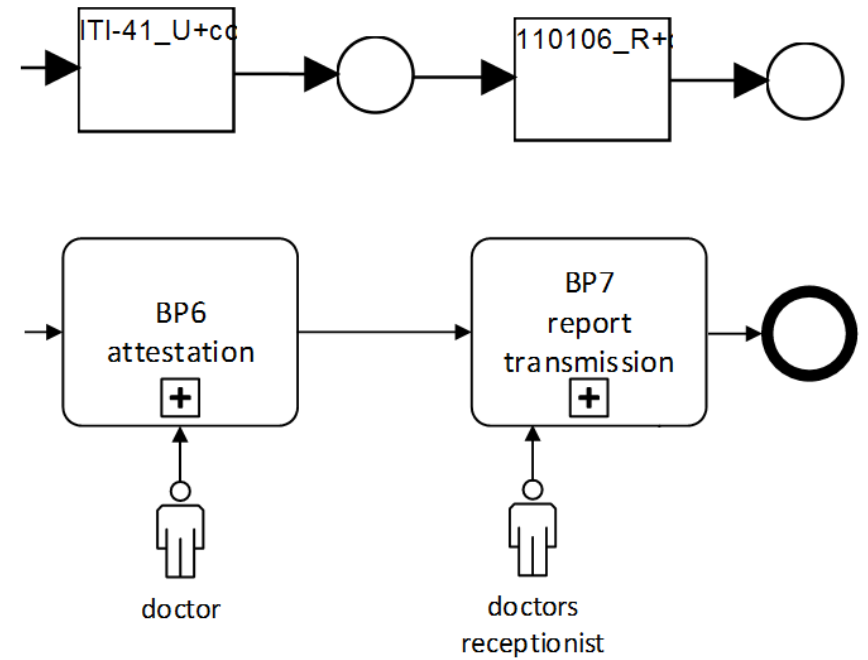

Fig. 7. The top part shows a detail of the reconstructed Petri net representing the last two transitions ITI-41_U and 110106_R. The lower part shows the respective part of the organizational workflow.

an IHE compliant environment also implement the Consistent Time (CT) integration profile that defines mechanisms to synchronize the time base between multiple actors and computers with a median error less than 1 second [27].

\section{B. Transformation Issues}

The identification of traces is a difficult task. As described in $\mathrm{V}$-A there is a lack of explicit notations of process instances and activities. In our simplified case we handled this by assigning the traces based on the patient identifier. This becomes a problem if the same patient visits the radiologist multiple times. Hence, in real-world audit logs a preprocessing step is needed to identify and mark the traces, e.g. by a combination of patient identifiers and timestamps.

Another issue with the transformation is the mapping. Based on the descriptions of the two meta-models, RFC-3881 and the XES standard extensions, the best semantic match was used. For further use this approach must be supported by the respective standardization organizations to avoid incorrect mappings.

\section{Further Steps}

Further research regarding the applicability of real-world ATNA logs for process mining is needed. A very important IHE integration profile, the Scheduled Workflow (SWF) profile, describes the interaction between actors in the radiology domain. In our test case we only recorded events regarding the organizational workflow of a radiological practice. SWF would enable more in-detail analysis of the processes taking place because the profile covers all patient data related workflows within the practitioners office.

\section{REFERENCES}

[1] W. Van der Aalst, A. Adriansyah, A. K. A. de Medeiros, F. Arcieri, T. Baier, T. Blickle, J. C. Bose, P. van den Brand, R. Brandtjen, J. Buijs et al., "Process mining manifesto," in Business process management workshops. Springer, 2012, pp. 169-194.
[2] W. Van der Aalst, Process Mining: Discovery, Conformance and Enhancement of Business Processes. Springer, 2011. [Online]. Available: http://books.google.at/books?id=I1 KOAfiqfxYC

[3] H. Verbeek, J. C. Buijs, B. F. Van Dongen, and W. M. Van Der Aalst, "Xes, xesame, and prom 6," in Information Systems Evolution. Springer, 2011, pp. 60-75.

[4] Fluxicon, "Discover your processes." 2015, http://fluxicon.com/disco/ Accessed: 2015-01-26.

[5] M. Lang, T. Bürkle, S. Laumann, and H.-U. Prokosch, "Process mining for clinical workflows: challenges and current limitations." Studies in health technology and informatics, vol. 136, pp. 229-234, 2007.

[6] R. Mans, H. Schonenberg, G. Leonardi, S. Panzarasa, A. Cavallini, S. Quaglini, and W. van der AALST, "Process mining techniques: an application to stroke care," Studies in health technology and informatics, vol. 136, p. 573, 2008

[7] R. Mans, M. Schonenberg, M. Song, W. M. van der Aalst, and P. J. Bakker, "Application of process mining in healthcare-a case study in a dutch hospital," in Biomedical Engineering Systems and Technologies. Springer, 2009, pp. 425-438

[8] Á. Rebuge and D. R. Ferreira, "Business process analysis in healthcare environments: A methodology based on process mining," Information Systems, vol. 37, no. 2, pp. 99-116, 2012.

[9] L. Perimal-Lewis, S. Qin, C. Thompson, and P. Hakendorf, "Gaining insight from patient journey data using a process-oriented analysis approach," in Proceedings of the Fifth Australasian Workshop on Health Informatics and Knowledge Management-Volume 129. Australian Computer Society, Inc., 2012, pp. 59-66.

[10] E. L. Siegel and D. S. Channin, "Integrating the healthcare enterprise: A primer: Part 1. introduction 1," Radiographics, vol. 21, no. 5, pp. 1339-1341, 2001.

[11] I. IHE, "Integrating the Healthcare Enterprise official web page, Member Organizations," 2015, www.ihe.net Accessed: 2015-01-26.

[12] E. GmbH, "ELGA - die elektronische Gesundheitsakte: Technische Grundlagen," 2015, http://www.elga.gv.at/index.php?id=24 Accessed: 2015-01-26.

[13] epSOS, "European Patients Smart Open Services: Technical Background," 2015, http://www.epsos.eu/technical-background/systemsstandards.html Accessed: 2015-01-26.

[14] TrilliumBridge, "TrilliumBridge: Bridging Patient Summaries across the Atlantic," 2015, http://www.trilliumbridge.eu/ Accessed: 2015-01-26.

[15] IHE, "Audit trail and node authentication (atna), ihe it infrastructure (iti) technical framework volume 1 (iti-tf-1) integration profiles," pp. 68-81, 2014.

[16] DICOM, "PS3.15 2014a - Security and System Management Profiles," 2014, http://medical.nema.org/standard.html Accessed: 2015-01-26.

[17] M. Bozkaya, J. Gabriels, and J. Werf, "Process diagnostics: a method based on process mining," in Information, Process, and Knowledge Management, 2009. eKNOW'09. International Conference on. IEEE, 2009, pp. 22-27

[18] G. Marshall, "Rfc 3881-security audit and access accountability message $\mathrm{xml}$ data definitions for healthcare applications. in," Request for Comments, vol. 3881, 2004.

[19] C. W. Günther and H. Verbeek, "Xes-standard definition," 2014

[20] F. Paster and E. Helm, "From ihe audit trails to xes event logs facilitating process mining," in MIE 2015; 27rd Int. Congress Eur. Federation Medical Informatics (Madrid, Spain), to appear 2015. IOS Press, 2015.

[21] O. Object Management Group, "Meta object facility (mof) core specification," 2014, www.omg.org/spec/MOF/2.4.2 Accessed: 2015-01-26.

[22] B. Elvesæter, A. Hahn, A.-J. Berre, and T. Neple, "Towards an interoperability framework for model-driven development of software systems," in Interoperability of enterprise software and applications. Springer, 2006, pp. 409-420.

[23] O. H. Tools, "The IHE Profiles Charter Project," 2015, www.openhealthtools.org Accessed: 2015-01-26.

[24] W. Van der Aalst, T. Weijters, and L. Maruster, "Workflow mining: Discovering process models from event logs," Knowledge and Data Engineering, IEEE Transactions on, vol. 16, no. 9, pp. 1128-1142, 2004

[25] R. S. Mans, W. M. van der Aalst, R. J. Vanwersch, and A. J. Moleman, "Process mining in healthcare: Data challenges when answering frequently posed questions," in Process Support and Knowledge Representation in Health Care. Springer, 2013, pp. 140-153.

[26] R. Cruz-Correia, I. Boldt, L. Lapão, C. Santos-Pereira, P. P. Rodrigues, A. M. Ferreira, and A. Freitas, "Analysis of the quality of hospital information systems audit trails," BMC medical informatics and decision making, vol. 13, no. 1, p. 84, 2013.

[27] IHE, "Consistent time (ct), ihe it infrastructure (iti) technical framework volume 1 (iti-tf-1) integration profiles," pp. 59-61, 2014. 